\title{
Vibration Analysis of Driving-Point System with Uncertainties Using Polynomial Chaos Expansion
}

\author{
Bin Xiao $\mathbb{D}^{1},{ }^{1}$ Yu-long Zhou, ${ }^{1}$ Chao Gao ${ }^{1},{ }^{1}$ Shuang-Xia Shi, ${ }^{1}$ Ze-Xi Sun, \\ and Zong-biao Song ${ }^{1,2}$ \\ ${ }^{1}$ School of Energy and Power Engineering, Northeast Electric Power University, Jilin City, Jilin 132012, China \\ ${ }^{2}$ Binzhou Special Equipment Inspection and Research Institute, Binzhou, Shandong 256600, China \\ Correspondence should be addressed to Bin Xiao; dr.binxiao@yahoo.com and Chao Gao; eriagao@163.com
}

Received 11 November 2019; Revised 15 July 2020; Accepted 28 July 2020; Published 28 September 2020

Academic Editor: Evgeny Petrov

Copyright (C) 2020 Bin Xiao et al. This is an open access article distributed under the Creative Commons Attribution License, which permits unrestricted use, distribution, and reproduction in any medium, provided the original work is properly cited.

\begin{abstract}
A vibration transfer analysis method based on polynomial chaos expansion (PCE) is proposed in this study and is used to analyze the stochastic dynamic compliance of uncertain systems with the Gaussian distribution. The random dynamic compliance is established by utilizing mode superposition on the system as the parameters of system uncertainties are regarded as input variables. Considering the asymptotic probability density function of mode shape, the dynamic compliance is decomposed into the mean of mode shape and the subsystem represented as an orthogonal polynomial expansion. Following this, the vibration transmission analysis approach is proposed for the random vibration. Results of a numerical simulation carried out employing the PCE approach show that broad-band spectrum analysis is more effective than narrow-band spectrum analysis because the former jump of the dynamic compliance amplitude is weakened. This proposed approach is valid and feasible, but since broad-band spectrum analysis loses some important information about the random vibration, both the aforementioned processes need to simultaneously be applied to analyze the random vibration transmission of low-medium frequency systems.
\end{abstract}

\section{Introduction}

The research of random dynamic system uncertainty originated from the stochastic finite element method (SFEM) applies the Monte Carlo simulation (MCS) method to the structural finite element analysis (FEA) process and repeats FEA to the model through specific samples $[1,2]$; consequently, the characteristics of the statistical distribution of the random response are obtained. For determined structural dynamic systems, extensive research regarding vibration analysis has been carried out, such as decoupling analysis on the nonlinear system $[3,4]$, analysis on vibration characteristics and response of structures, and study on the vibration transmission path.

Furthermore, the roles of various boundary conditions $[5,6]$, for example, classical restraints, elastic supports and their combinations, and geometric properties [7], such as, cylindrical, conical, and spherical, have been taken into account in the research process. It is thus of great interest to understand thoroughly the vibration behaviors of the system. After more accurate and applicable methods for determined vibration analysis are developed, perturbation theory is applied to the transformation equation, including stochastic distribution parameters, stochastic elastic boundary support structures, and stochastic boundary condition problems, and then the stochastic problem is transformed into a series of deterministic differential equations and boundary conditions, and finally, the random vibration analysis is realized. Furthermore, in order to obtain the mean and standard deviations of the results, the uncertainty of parameters and nonparameters should be considered. Fortunately, the stochastic perturbation method is devoted to the theoretical aspects and computational implementation of the generalized stochastic perturbation technique. It is based on any-order Taylor expansions of random variables and enables for determination of up to fourth-order probabilistic moments and characteristics of the physical system response, which provides grounding in the basic elements of statistics and probability and reliability engineering [8]. Therefore, Huang et al. [9] obtained the 
approximate statistical expressions of stochastic eigenvalues. $\mathrm{Wu}$ and Law [10] discussed the statistical prediction of the dynamic response of bridge structures under random moving loads. Xiao et al. [11] analyzed the random vibration characteristics of the Levinson beam on random elastic constraint conditions. Szafran et al. [12] presented a reliability estimation procedure for steel lattice telecommunication towers based on tensioned joint reliability and used the first-order and second-order reliability methods to confirm the elastic-plastic range of joint reliability. Handa and Anderson [13] used the first- and second-order perturbation of the mean of stochastic variables to obtain statistical properties of the response when analyzing the fluctuation of stochastic variables in the system. On this basis, Chen et al. [14] proposed interval variables to describe various uncertain parameters of the system. Adhikari [15] presented variable matrix distributions of the mass, stiffness, and damping of the quantitative linear structure dynamics under uncertainty based on the optimal stochastic matrix theory [16]. Typically, the statistics of the system are obtained via MCS, SFEM, or polynomial chaos expansion (PCE). MCS is often used to obtain reference results. Although simulation techniques can be used for a wide range of structural dynamics problems, it is computationally intractable, particularly for large-scale problems. It is equally evident that while numerical techniques based on Taylor series expansion are efficient, they do not yield sufficient accuracy when there are significant uncertainties.

Due to its accuracy and ability to provide powerful representations of vibration transfer capabilities of nonembedded PCE technology, it has become increasingly popular among researchers and has evolved into an attractive approach. Within the framework of orthogonal polynomial expansion, Pascual and Adhikari [17] proposed four kinds of solution forms: Rayleigh quotient, power method, inverse power method, and eigenvalue equation based on the mixed perturbation PCE method. Sarsri et al. [18] obtained the first two statistical moments of the frequency transfer function through chaos expansion and elaborated the coupling problems of the first- and secondorder PCE; furthermore, more than three order statistics of the structural response were analyzed by Xiao and Li [19]. Sepahvand et al. [20] used the truncated PCE of arbitrary random basis to express the uncertain elastic modulus, eigenfrequency, and mode of an orthotropic plate in order to study the stochastic free vibration of the plate. Bahmyari et al. [21] combined the meshless Galerkin method with the generalized PCE to analyze the stochastic bending of moderately thick plates with elastic constraint edges. Najlawi et al. $[22,23]$ proposed a hybrid multiobjective imperialist competitive algorithm (MOICA) and MCS method for the multiobjective robust design optimization of a mechanism and further developed a hybrid MOICA-PCE algorithm to be exploited for robust optimization [24]. In addition, Hadigol and Doostan [25] presented a hybrid sampling method that employs the so-called alphabetic optimality criteria used in the context of the optimal design of experiments in conjunction with coherence-optimal samples, especially for high-order PCE.
The main objective of this study was to present an efficient and reliable method for evaluating the vibration transmission and response of a random dynamical system based on PCE of the dynamic compliance when subjected to pure harmonic excitation. For this purpose, mode superposition-based dynamic compliance is introduced for the random dynamical system, which is used in extracting the mean mode when considering random mode elements with the log-normal asymptotic distribution. A new form of PCE is proposed to give the approximate expression of the dynamic compliance, which is combined with embedded Fourier-Hermite polynomial expansion. Thereafter, the proposed approach is established for discussing the vibration transmission and response of the random vibration system.

This paper is organized as follows. Section 2 briefly reviews the dynamic compliance and orthogonal polynomial expansion theory; thereafter, the PCE approach is presented. In Section 3, numerical simulation is used to demonstrate the PCE approach on both processes of broad-band spectrum analysis and narrow-band spectrum analysis, and analysis of random vibration transmission and response is implemented by comparing with MCS. Finally, in Section 4, the concluding remarks are given.

\section{Theoretical Method}

2.1. Dynamic Compliance. Let us consider a multidegree-offreedom linear structural system with mass, damping, and stiffness matrices $\mathbf{M}, \mathbf{C}$, and $\mathbf{K}$, respectively. The equations of motion describing the forced vibration of a linear and damped discrete system are

$$
\mathbf{M} \frac{\partial^{2} \mathbf{Y}}{\partial t}+\mathbf{C} \frac{\partial \mathbf{Y}}{\partial t}+\mathbf{K Y}=\mathbf{F}
$$

where $\mathbf{Y}$ is the nodal displacement vector and $\mathbf{F}$ is the external harmonic excitation with angular frequency $\omega$.

An undamped or proportional damped system is considered in this study, whose matrices $\mathbf{M}, \mathbf{C}$, and $\mathbf{K}$ are real symmetric. Hence, the dynamic compliance matrix based on the mode superposition is defined by

$$
\left.\{\rho\}_{i j}\right|_{\substack{i: x \\ j: y}}=\sum_{n=1}^{\infty} \frac{\phi_{n}(y) \phi_{n}(x)}{j \omega \widetilde{Z}_{n}},
$$

where $\{\rho\}_{i j}$ is the vibration transmission from the $j$ th node to the $i$ th node and $\widetilde{Z}_{n}$ [26] is the mode impedance:

$$
\widetilde{Z}_{n}=j \mathbf{M}_{n}\left(\omega-\frac{\omega_{n}^{2}}{\omega}\right)+\frac{\mathbf{M}_{n} \eta_{n} \omega_{n}^{2}}{\omega},
$$

where $\mathbf{M}_{n}, \phi_{n}(\cdot)$, and $\eta_{n}$ are the mode mass, mode shape, and mode damping ratio with respect to the $n$-th-order natural frequency $\omega_{n}$, respectively.

However, since we are interested in the limit as $\eta_{n} \longrightarrow 0$, consider $N_{e}$-order modal truncation; the real dynamic compliance of equation (2) for $x=y$, 


$$
\left.\{\rho\}_{i i}\right|_{i: x}=\sum_{n=1}^{N_{e}} \frac{\phi_{n}^{2}(x)}{j \omega \widetilde{Z}_{n}}
$$

may become arbitrarily large $\mid\{\rho\}_{i i} \|_{i: x} \longrightarrow+\infty$ only if the external excitation frequency $\omega$ becomes very close to some natural frequency $\omega_{n}$.

The weight of eigenfunctions,

$$
w=\phi_{n}^{2}(x),
$$

is defined on $0 \leq w \leq 1$. The eigenvector of the matrix, $\phi_{n}(x)$, at driving point $x$ has to satisfy the orthogonality of eigenfunctions. A convenient way to characterize the statistics of $w$ is via the moments of arbitrary index $q>0$ :

$$
Y_{q}=\int_{0}^{1} \mathrm{~d} w \Pi_{N} w^{q} w,
$$

where $\Pi_{N}(\cdot)$ is the probability density function (PDF), and $\mathrm{N}$ is the dimension of the square matrices of the system. As a consequence, the weight $w$ is a random variable distributed in a Gaussian orthogonal ensemble (GOE) which obeys lognormal distribution $\prod_{N}$ [27]. The authors in [27] have given the correlation between the log-normal distribution and the multifractal algorithm as follows:

$$
\Pi_{N}(w)=\frac{1}{w \sqrt{2 \pi \sigma^{2} \ln N}} \mathrm{e}^{\left(\ln w+\alpha_{0} \ln N\right)^{2} / 2 \sigma^{2} \ln N}, \quad \alpha \equiv-\frac{\ln w}{\ln N} .
$$

Hence, when $\alpha=\alpha_{0}$, the equation holds: the spectrum of fractal dimensions $g\left(\alpha_{0}\right)=1 ; \bar{w}=\sqrt{w}$ satisfies probability distribution $\Pi_{N}(\bar{w})$, i.e.,

$$
\Pi_{N}(\bar{w})=\frac{2}{\bar{w} \sqrt{2 \pi \sigma^{2} \ln N}} e^{-\left(2 \ln \bar{w}+\alpha_{0} \ln N\right)^{2} / 2 \sigma^{2} \ln N} .
$$

If the external excitation frequency $\omega$ becomes very close to some natural frequency $\omega_{n}$, then the corresponding term dominates over the others in equation (4), and the dynamic compliance can be approximated by this biggest term; therefore, in such case, the probability distribution of the dynamic compliance is presented as follows:

$$
P\left(\left.\{\tilde{\rho}\}_{i i \mid}\right|_{i: x}\right)_{\left.\{\tilde{\rho}\}_{i i}\right|_{i: x} \longrightarrow+\infty}^{\simeq} \frac{\rho(\omega)}{\left(\left.\{\tilde{\rho}\}_{i i \mid}\right|_{i: x}\right)^{2}} .
$$

Otherwise, the dynamic compliance can be approximated by

$$
\begin{aligned}
\left.\{\widetilde{\rho}\}_{i i}\right|_{i: x} & \simeq Y_{1} \sum_{n=1}^{N_{e}} \frac{\phi_{n}^{2}(x)}{j \omega \widetilde{Z}_{n} \sqrt{\phi_{n}^{2}(x)}} \\
& \simeq Y_{1} \sum_{n=1}^{N_{e}} l_{n},
\end{aligned}
$$

where $Y_{1}$ is the mean of $\bar{w}$ when all the random variables are mean values [28], with $Y_{1}=E[\bar{w}] \cdot l_{n}$ being the polynomial function.

\subsection{Orthogonal Polynomial Expansion}

2.2.1. Fourier-Hermite Polynomials. Consider a continuous, differentiable, real-valued, multivariate function $y(\mathbf{x})$ that is defined on $\mathbf{x}=\left\{x_{1}, x_{2}, \ldots, x_{M}\right\}^{T} \in \mathbb{R}^{M}$, where $\mathbb{R}^{M}$ is an $M$ dimensional real vector space. A dimensional decomposition of $l_{n}=y(\mathbf{x})$, described by

$$
y(\mathbf{x})=y_{0}+\sum_{S=1}^{M} \sum_{1 \leq a_{1}<\cdots<a_{S} \leq M} y_{a_{1} \ldots a_{S}}\left(x_{a_{1}}, \ldots, x_{a_{S}}\right),
$$

can be viewed as a finite hierarchical expansion of an output function in terms of its input variables with increasing dimensions, where $y_{0}$ is a constant representing the mean response of the transfer function, and $y_{a_{1} \ldots a_{S}}\left(x_{a_{1}}, \ldots, x_{a_{S}}\right)$ is an $S$-variate component function quantifying the cooperative effects of $S$ input variables $x_{a_{1}}, \ldots, x_{a_{s}}$.

In order to generate a hierarchical and convergent sequence of approximations of $y(\mathbf{x})$, considering input variables following I.I.D Gaussian distribution, a general Svariate approximation of $y(\mathbf{x})$ is obtained [29]:

$$
\tilde{y}_{S}(\mathbf{x}) \simeq y_{0}+\sum_{S=1}^{M} \sum_{1 \leq a_{1}<\ldots<a_{S} \leq M} \sum_{b_{S}=1}^{m} \ldots \sum_{b_{1}=1}^{m} C_{a_{1} \ldots a_{S} b_{1} \ldots b_{S}} \prod_{k=1}^{S} \psi_{b_{k}}\left(x_{a_{k}}\right),
$$

where

$$
\begin{aligned}
C_{a_{1} \ldots a_{s} b_{1} \ldots b_{s}} & =\int_{A^{s}} y_{a_{1} \ldots a_{s}}\left(x a_{1}, \ldots, x_{a_{S}}\right) \prod_{k=1}^{S} \psi_{b_{k}}\left(x_{a_{k}}\right) f_{k}\left(x_{k}\right) d x_{k} \\
& =\int_{A^{M}} y_{(x)} \prod_{k=1}^{S} \psi_{b_{k}}\left(x a_{k}\right) f_{X}(x) d x
\end{aligned}
$$


is a coefficient associated with the product of $b_{1}$ through $b_{S}$ basis functions expressed in terms of $x_{a_{1}}, \ldots, x_{a_{s}}$, where $x_{k}$ follows probability density function (PDF) $f_{k}\left(x_{k}\right), X$ follows the joint PDF $f_{\mathbf{X}}(\mathbf{x})$, and $\psi_{b}\left(x_{a}\right)$ is the $b$-th order Hermite polynomial, where $b \leq m$.

2.2.2. Dimension-Reduction Integration for Calculating Coefficients. Following an early idea by $\mathrm{Xu}$ and Rahman
[30], a lower-variate approximation of the $M$-variate function $y(\mathbf{x})$ is considered, which leads to a reduction in the dimensionality of the $M$-dimensional integral coefficients of the equation. Using Xu and Rahman's multivariate function theorem [30], it can be shown that a special $R$ variate approximation of $y(\mathbf{x})$, defined by

$$
y_{R}(\mathbf{x})=\sum_{k=0}^{R}(-1)^{k}\left(\begin{array}{c}
M-R+k-1 \\
k
\end{array}\right) \sum_{1 \leq k_{1}<\cdots<k_{R-k} \leq M} y\left(x_{k_{1}}, x_{k_{2}}, \ldots, x_{k_{R-k}}\right)
$$

consists of all terms of the Taylor series of $y(\mathbf{x})$ that have less than or equal to $R$ variables, where $y\left(x_{k_{1}}, x_{k_{2}}, \ldots, x_{k_{R-k}}\right)$ represents the $(R-k)$ th dimensional component function of $y(\mathbf{x})$, with $R<M$ and $k=0, \ldots, R$.
Substituting equation (14) into equation (13), the polynomial expansion coefficients become

$$
C_{a_{1} \ldots a_{S} b_{1} \ldots b_{S}} \simeq \sum_{k=0}^{R}(-1)^{k}\left(\begin{array}{c}
M-R+k-1 \\
k
\end{array}\right)_{k_{1}, \ldots, k_{R-k}=1 ; k_{1}<\cdots<k_{R-k}}^{M} \int_{A^{R-k}} y\left(x_{k_{1}}, x_{k_{2}}, \ldots, x_{k_{R-k}}\right) \prod_{m=1}^{S} \psi_{b_{m}}\left(x_{a_{m}}\right) \prod_{m=1}^{R-k} f_{k_{m}}\left(x_{k_{m}}\right) d x_{k_{m}},
$$

and the mean is

$$
y_{0} \simeq \sum_{k=0}^{R}(-1)^{k}\left(\begin{array}{c}
M-R+k-1 \\
k
\end{array}\right)_{k_{1}, \ldots, k_{R-k}=1 ; k_{1}<\cdots<k_{R-k}}^{M} \int_{A^{R-k}} y\left(x_{k_{1}}, x_{k_{2}}, \ldots, x_{k_{R-k}}\right) \prod_{m=1}^{R-k} f_{k_{m}}\left(x_{k_{m}}\right) d x_{k_{m}} .
$$

It is obvious that introducing the dimension-reduction integration for calculating coefficients is substantially simpler and more efficient than performing one $M$-dimensional integral, particularly when $R \ll M$.
2.2.3. Gauss-Hermite Integration. The Gauss-Hermite multiple quadrature formulas for Hermite polynomials are

$$
\int_{-\infty}^{+\infty} \ldots \int_{-\infty}^{+\infty} f\left(x_{a_{1}}, \ldots, x_{a_{S}}\right) e^{-\sum_{k=1}^{S} x_{k}^{2}} \prod_{k=1}^{S} d x_{k}=\sum_{a_{S}=1}^{Q_{S}} \ldots \sum_{a_{1}=1}^{Q_{1}} \prod_{k=1}^{S} W_{k} f\left(x_{a_{1}}, \ldots, x_{a_{S}}\right)
$$

where $Q_{1}, \ldots, Q_{S}$ are the integral points and associated coefficient $W_{k}$ depends on the probability distribution $f\left(x_{a_{1}}, \ldots, x_{a_{S}}\right)$ of $x_{a_{1}}, \ldots, x_{a_{S}}$. Considering a special term $e^{-x^{2}}$, equation (17) can be transformed into the summation form and used to solve the polynomial coefficients and mean values efficiently. Correspondingly, in order to improve its calculation accuracy and efficiency, we can define $Q=Q_{1}=\cdots Q_{S}$.

\subsection{Error Analysis}

2.3.1. Mean. For the driving-point vibration analysis, i.e., $x=y$, the expectation of the dynamic compliance in equation (4) is obtained:

$\mathbf{E}\left[\left.\{\rho\}_{i i}\right|_{i: x}\right]=\mathbf{E}\left[\sum_{n=1}^{N_{e}} \frac{\phi_{n}^{2}(x)}{j \omega \widetilde{Z}_{n}}\right]=\sum_{n=1}^{N_{e}} \mathbf{E}\left[\frac{1}{j \omega \widetilde{Z}_{n}}\right] \mathbf{E}\left[\phi_{n}^{2}(x)\right]$. 
And the expectation of the dynamic compliance in equation (9) is

$$
\begin{aligned}
\mathbf{E}\left[\left.\{\widetilde{\rho}\}_{i i}\right|_{i: x}\right] & \simeq Y_{1} \cdot \mathbf{E}\left[\sum_{n=1}^{N_{e}} \frac{\phi_{n}^{2}(x)}{j \omega \widetilde{Z}_{n} \sqrt{\left|\phi_{n}^{2}(x)\right|}}\right] \\
& \simeq Y_{1} \sum_{n=1}^{N_{e}} \mathbf{E}\left[\frac{1}{j \omega \widetilde{Z}_{n}}\right] \mathbf{E} \frac{\phi_{n}^{2}(x)}{\sqrt{\left|\phi_{n}^{2}(x)\right|}} .
\end{aligned}
$$

Therefore, equation (19) becomes

$$
\mathbf{E}\left[\left.\{\widetilde{\rho}\}_{i i}\right|_{i: x}\right]=\sum_{n, m=1}^{N_{e}} \mathbf{E}\left[\frac{1}{j \omega \widetilde{Z}_{n}}\right] \mathbf{E}\left[\phi_{n}(x) \phi_{m}(x)\right]
$$

Comparing equation (19) with equation (20) and considering the relationship $\sum_{n=1}^{N_{e}} \phi_{n}^{2}(x) \simeq\left(\sum_{n=1}^{N_{e}} \phi_{n}^{2}(x)\right)^{2}$, the following relation can be obtained:

$$
\mathbf{E}\left[\left.\{\widetilde{\rho}\}_{i i}\right|_{i: x}\right]=\mathbf{E}\left[\left.\{\rho\}_{i i}\right|_{i: x}\right] .
$$

2.3.2. Standard Error. In the statistical analysis procedure of random variables $\left\{x_{1}, x_{2}, \ldots, x_{m}\right\}$, the standard error (SE) is defined as [18]

$$
D_{r . m . s}[x]=\sqrt{\frac{1}{m} \sum_{j=1}^{m}\left(x_{j}-\mathbf{E}\left[x_{j}\right]\right)^{2} .}
$$

Supposing a quantity is a function of the measured variables, i.e., $y=f\left(x_{1}, x_{2}, \ldots, x_{m}\right)$, the error propagation equation is obtained as follows:

$$
D_{r . m . s}^{2}[y]=\sum_{j=1}^{m}\left(\frac{\partial f}{\partial x_{j}} D_{r . m . s}\left[x_{j}\right]\right)^{2} \text {. }
$$

By using equations (23) and (24), the SE of the dynamic compliance in equation (4) can be obtained:

$$
D_{r . m . s}^{2}\left[\left.\{\rho\}_{i i}\right|_{i: \mathrm{x}}\right]=\sum_{n=1}^{N_{e}}\left\{\left(\frac{2}{j \omega \widetilde{Z}_{n}}\right)^{2} \phi_{n}^{2}(x) D_{r . m . s}^{2}\left[\phi_{n}(x)\right]+\left(\frac{-1}{j \omega \widetilde{Z}_{n}^{2}}\right)^{2} \phi_{n}^{4}(x) D_{r . m . s}^{2}\left[\widetilde{Z}_{n}\right]\right\}
$$

And then, the SE of dynamic compliance (10) can be obtained as follows:

$$
D_{r . m . s}^{2}\left[\left.\{\widetilde{\rho}\}_{i i}\right|_{i: x}\right]=\sum_{n=1}^{N_{e}}\left\{\left(\frac{1}{j \omega \widetilde{Z}_{n}}\right)^{2}\left(\frac{\phi_{n}^{2}(x)}{2}\right)\left(\frac{Y_{1}}{\omega}+\frac{\partial Y_{1}}{\partial \varpi}\right) D_{r . m . s}^{2}\left[\phi_{n}(x)\right]+\left(\frac{-\omega}{j \omega \widetilde{Z}_{n}^{2}}\right)^{2} Y_{1}^{2} D_{r . m . s}^{2}\left[\widetilde{Z}_{n}\right]\right\} .
$$

Using equations (24) and (25), the SE level can be defined as

$$
\begin{aligned}
e_{L_{d}} & =10 \lg D_{r . m . s}^{2}\left[\left.\{\rho\}_{i i}\right|_{i: x}\right], \\
\tilde{e}_{L_{d}} & =10 \lg D_{r . m . s}^{2}\left[\left.\{\tilde{\rho}\}_{i i}\right|_{i: x}\right] .
\end{aligned}
$$

Then, the statistic of the PCE-based dynamic compliance can be yielded as the format of the SE level, i.e.,

$$
\Delta e_{L_{d}}=\widetilde{e}_{L_{d}}-e_{L_{d}}=10 \lg \frac{D_{r . m . s}^{2}\left[\left.\{\tilde{\rho}\}_{i i}\right|_{i: x}\right]}{D_{r . m . s}^{2}\left[\left.\{\rho\}_{i i}\right|_{i: x}\right]} .
$$

It follows that the proposed PCE approach is reasonable and feasible, by equation (21), but its error in equation (27) is inevitable, according to equations (24) and (25). So, the curve shape distortion (CSD) is defined based on equation (21) as

$$
\mathrm{CSD}=\int_{-\infty}^{+\infty}\left|p_{\mathrm{MCS}}(x)-p_{\mathrm{PCE}}(x)\right| \mathrm{d} x,
$$

where $p_{\mathrm{MCS}}(x)$ and $p_{\mathrm{PCE}}(x)$ are the PDFs of the SE level by employing MCS and PCE.

\section{Numerical Simulation}

3.1. FEM-Based Plate Model. Rectangular plates are widely used in our daily life; all kinds of modeling method have been proposed for the vibration characteristics of rectangular plates with system certainty [31]. In this paper, a rectangular plate is fixed at its left boundary and supported by springs at its right boundary. Considering the vibration transmission and response at a driving point, the coordinate of which is $(0.8,0.4)$, with single-point harmonic excitation, FEM modeling can be given, shown in Figure 1 with parameters listed in Table 1 . The stiffness $K_{i}$ is the $i$-th random variable with a total of $n=19$, that is, $K_{i} \sim N\left(\mu, \sigma^{2}\right), \quad i=1,2, \ldots, n$, follows the PDF $N(\cdot, \cdot)$ of a Gaussian distribution with $\mu=10000 / n$ and $\sigma=2500 / n$.

In order to demonstrate the efficiency of PCE, some benchmark tests are analyzed with random parameters and vertical vibration. The proposed PCE approach with 


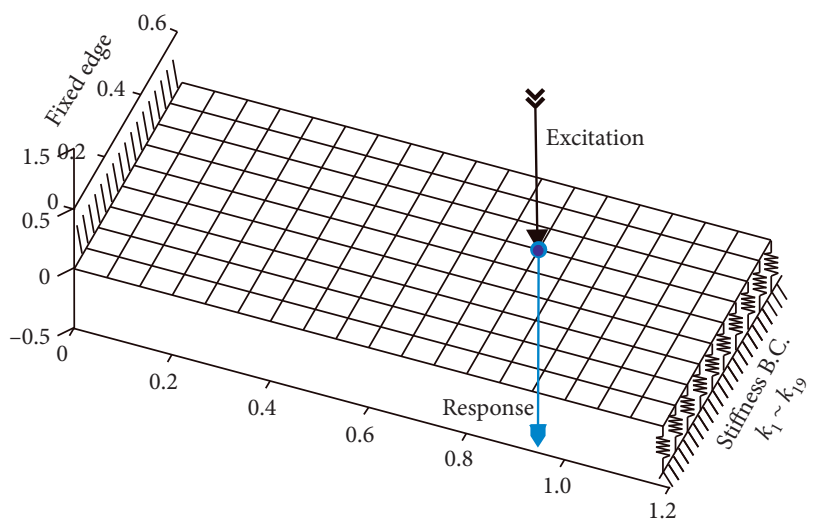

FIGURE 1: Schematic of FEM modeling of the rectangular plate.

Table 1: Parameters and random boundary conditions of the rectangular plate.

\begin{tabular}{lccc}
\hline Variables & Distribution & Mean & Variation coefficient \\
\hline Young's modulus $E$ & Deterministic & $2 \times 10^{11} \mathrm{~N} / \mathrm{m}^{2}$ & - \\
Mass density $\rho$ & Deterministic & $7860 \mathrm{~kg} / \mathrm{m}^{3}$ & - \\
Poisson's ratio $\mu$ & Deterministic & 0.3 & - \\
Length $L$ & Deterministic & $1.2 \mathrm{~m}$ & - \\
Width $B$ & Deterministic & $0.6 \mathrm{~m}$ & - \\
Thickness $\delta$ & Deterministic & $7.5 \times 10^{-3} \mathrm{~m}$ & - \\
Spring stiffness $\sum_{i=1}^{n} K_{i}$ & Gaussian & $10 \mathrm{k} \mathrm{N} / \mathrm{m}$ & 0.25 \\
Damping coefficient $\eta_{\mathrm{n}}$ & Uniform & $0.5 \%$ & - \\
\hline
\end{tabular}

univariate $(S=1)$, bivariate $(S=2)$, and trivariate $(S=3)$ approximations is elaborated and considered to be the main results of this study. For the sake of assessing the accuracy of the PCE method, a comparison MCS approach is used, with its results using $1 \times 10^{5}$ samples, serving as reference results. The errors between the estimated and reference responses are defined in equation (27), and CSD is defined in equation (28).

\subsection{Narrow-Band Spectrum Analysis}

(a) Employing mode analysis, the natural frequencies within $[0,500 \mathrm{~Hz}]$ were obtained, as listed in Table 2. Assuming that random variables are mean values, we obtained the mean model of the dynamic compliance, as shown in Figure 2. For comparison purposes, the mean of the dynamic compliance is given in Figure 3. The curves in Figures 2 and 3 are almost identical; these results coincided with equation (21), i.e., the mean model embodies the mean of the vibration transmission and response of the random system, thus implying that the PCE approach is valid and feasible for narrow-band spectrum analysis. ,

Figure 4 shows the error analysis curves of the dynamic compliance spectral level. Results reveal that, for both univariate $(S=1)$ and bivariate $(S=2)$ approximations of the PCE approach, very small differences exist from the results from MCS. That is to say, the error of the PCE approach cannot be eliminated by using higher $S$-variate approximation, even if $S \longrightarrow N$. After a brief review of the PCE approach, it is not difficult to find that the majority of errors was caused by mode-shape asymptotic estimation, multirandom variable decoupling, and Hermite polynomial truncation in the orthogonal polynomial expansion. Fortunately, the maximum relative error is less than $3 \mathrm{~dB}$, so the statistical analysis results are excellent in the low-frequency band within $100 \mathrm{~Hz}$ and are still acceptable at frequencies more than $100 \mathrm{~Hz}$.

(b) In order to further discuss the detailed error of PCE, to consider, for example, just the two frequency bands of $18 \sim 22 \mathrm{~Hz}$ and $29 \sim 35 \mathrm{~Hz}$, the PDF curves of the dynamic compliance amplitude are plotted in Figures 5 and 6, respectively. The compared results indicate that there exists CSD, but it is very small difference between the estimated and reference results.

Although the stiffness of the spring follows the I.I.D. Gaussian distribution, it is evident that the PDF curves of the dynamic compliance do not obey the Gaussian distribution but embody a trend towards the Gaussian distribution. The dynamic compliance curves are in the damping control area, which are very sensitive to damping near the natural frequency. It can be seen from Figure 2 and Table 2 that the dominant mode of vertical vibration (resonant frequency) is not evident within the frequency band of $18 \sim 22 \mathrm{~Hz}$, but is evident within the frequency band of $29 \sim 35 \mathrm{~Hz}$. Correspondingly, a comparison of 


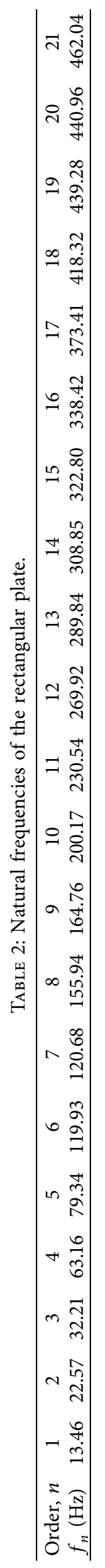




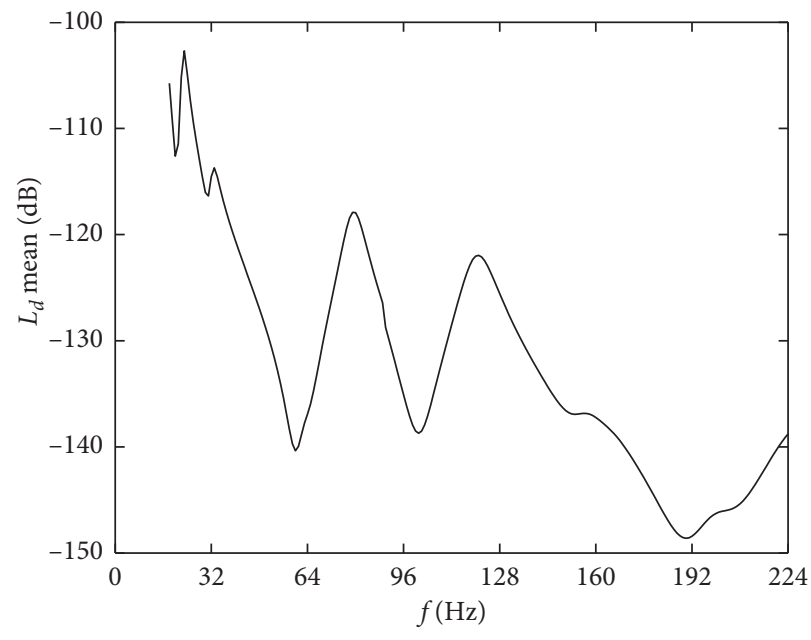

$-L_{d}$

Figure 2: Mean model of the dynamic compliance.

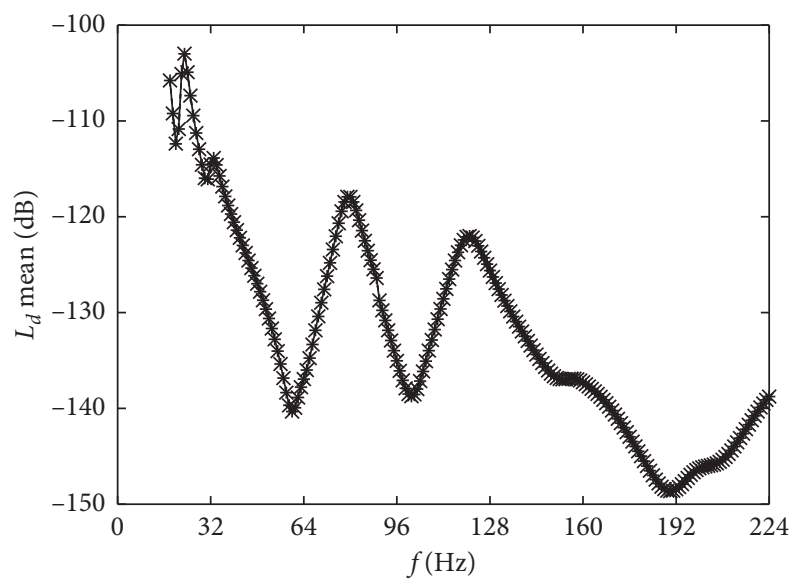

- MCS

--+ PCE, univariate

- $\times$ - PCE, bivariate

Figure 3: Mean of the dynamic compliance.

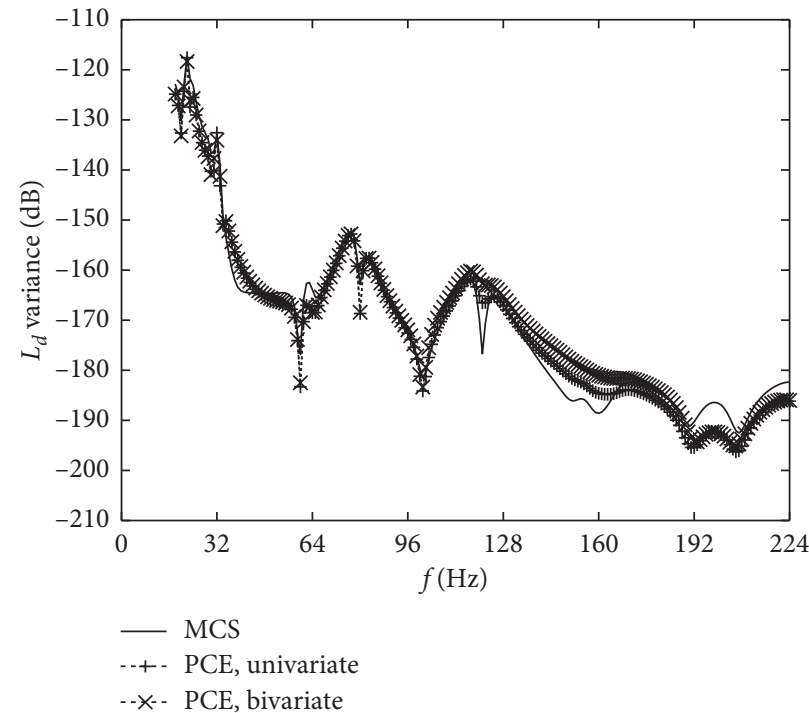

Figure 4: Error analysis curves of the dynamic compliance spectral level. 


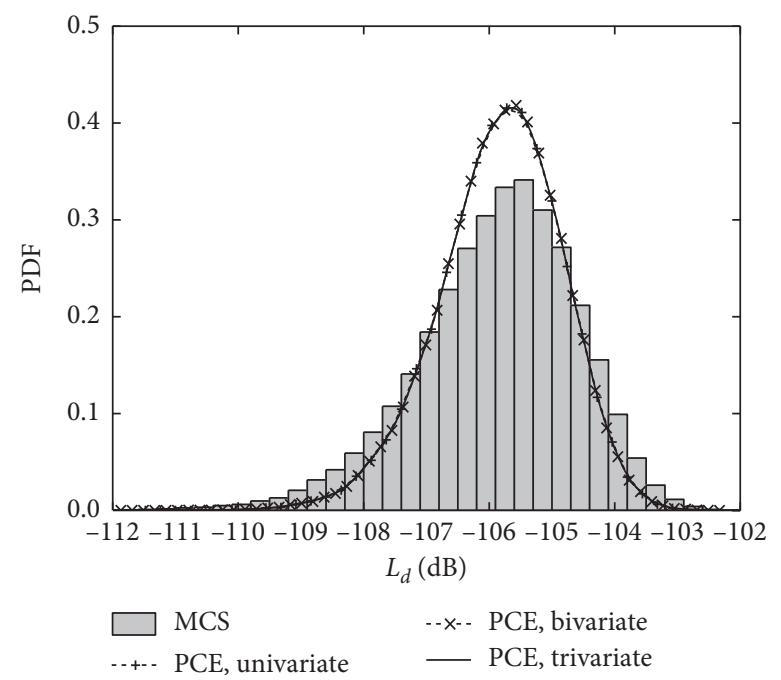

(a)

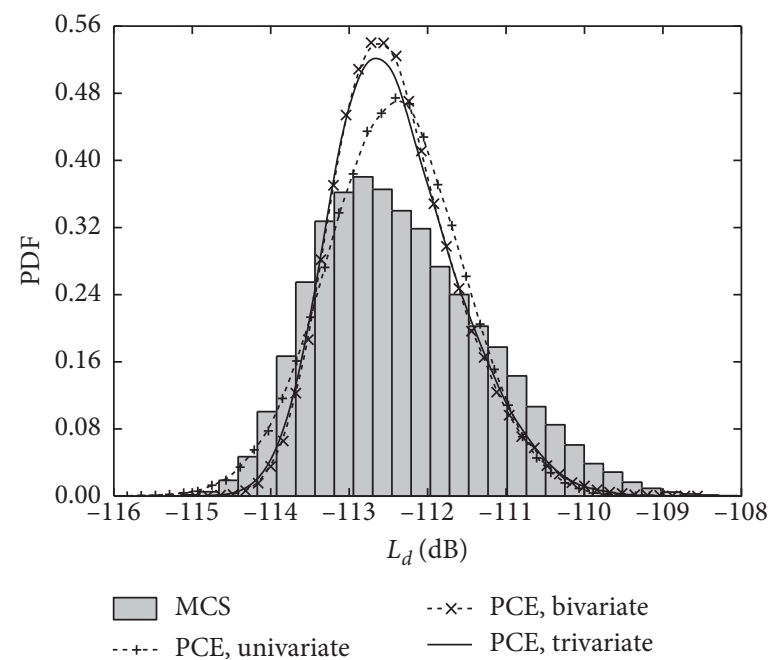

(c)

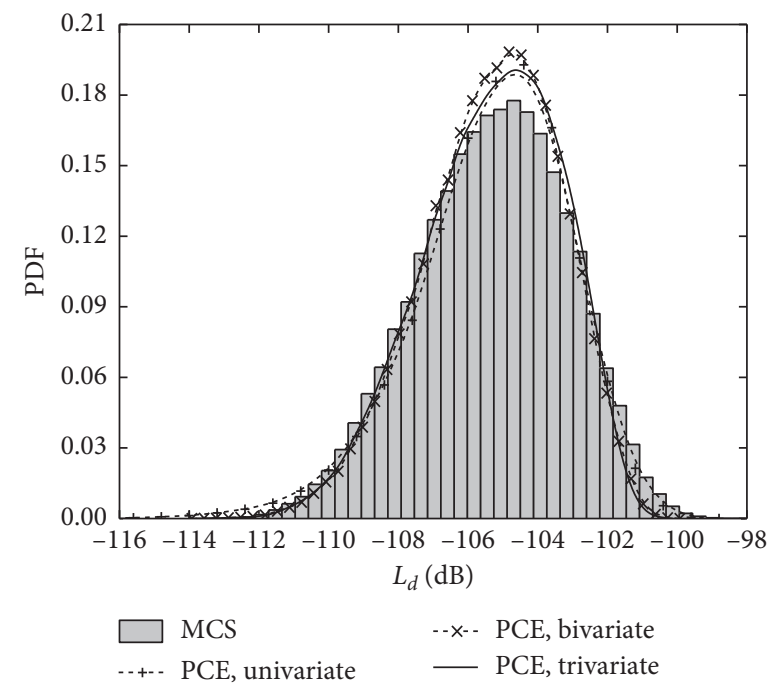

(e)

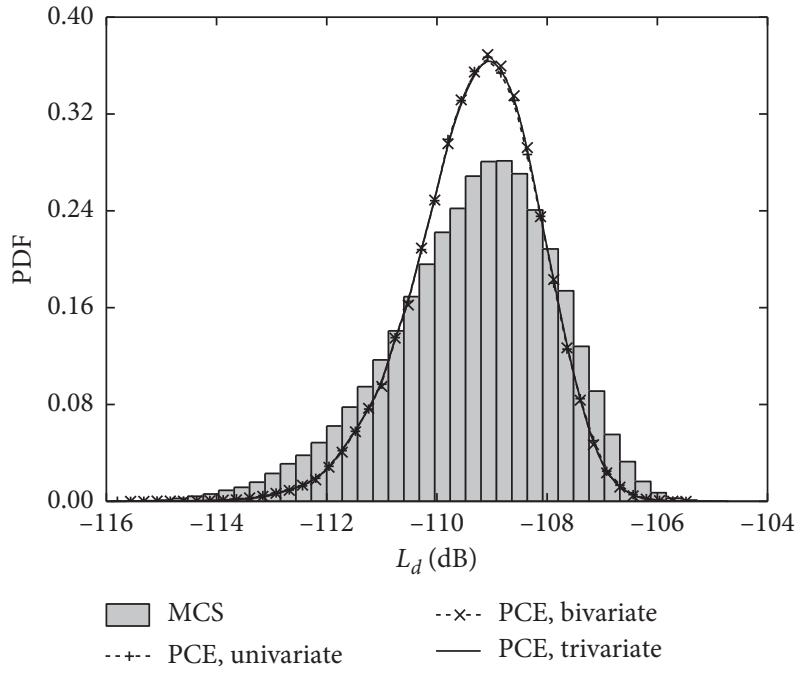

(b)

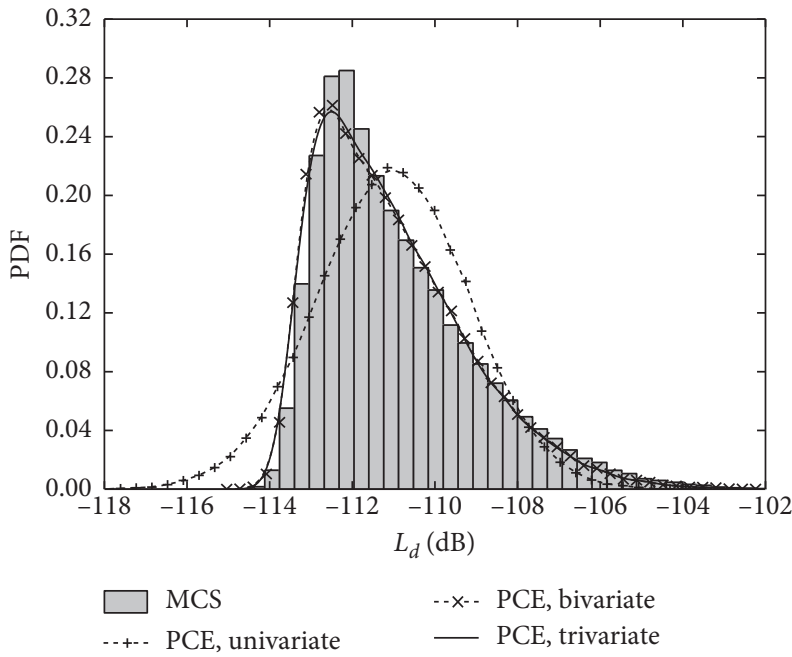

(d) 

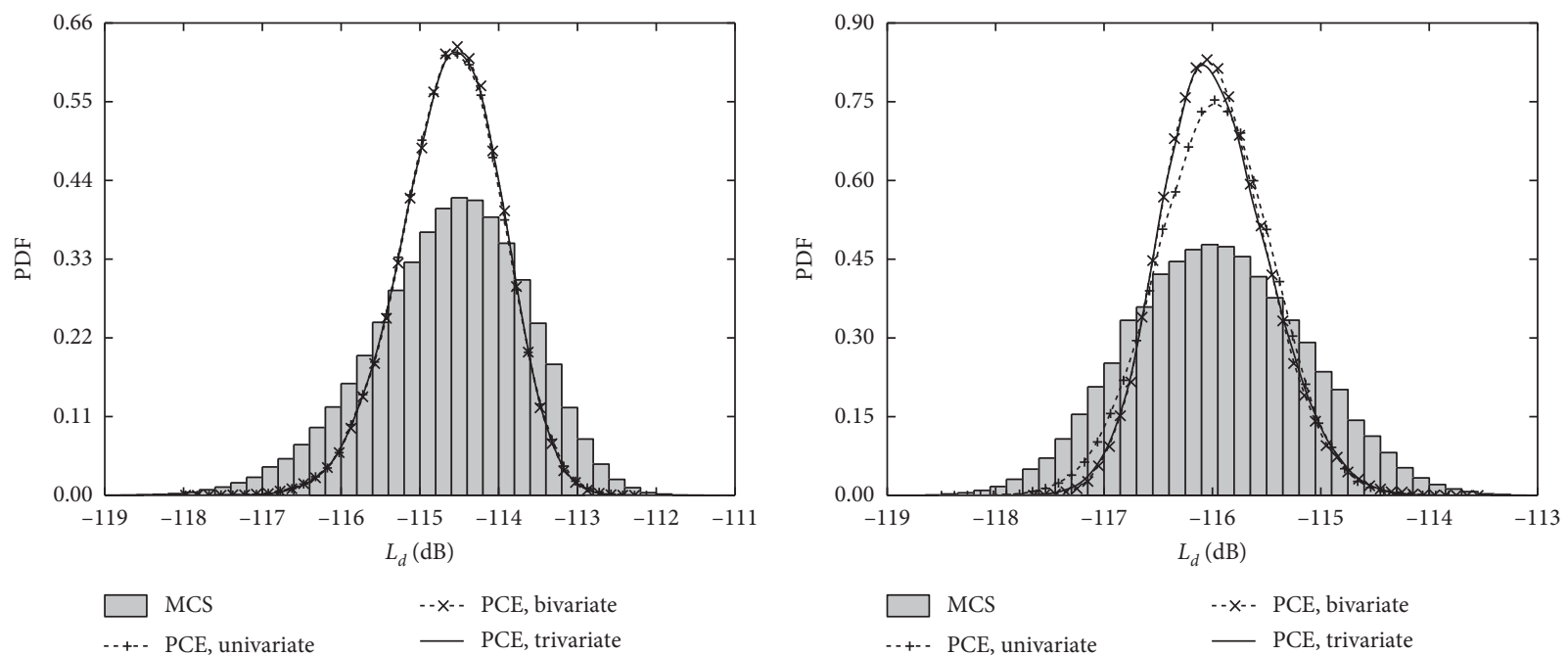

(a)
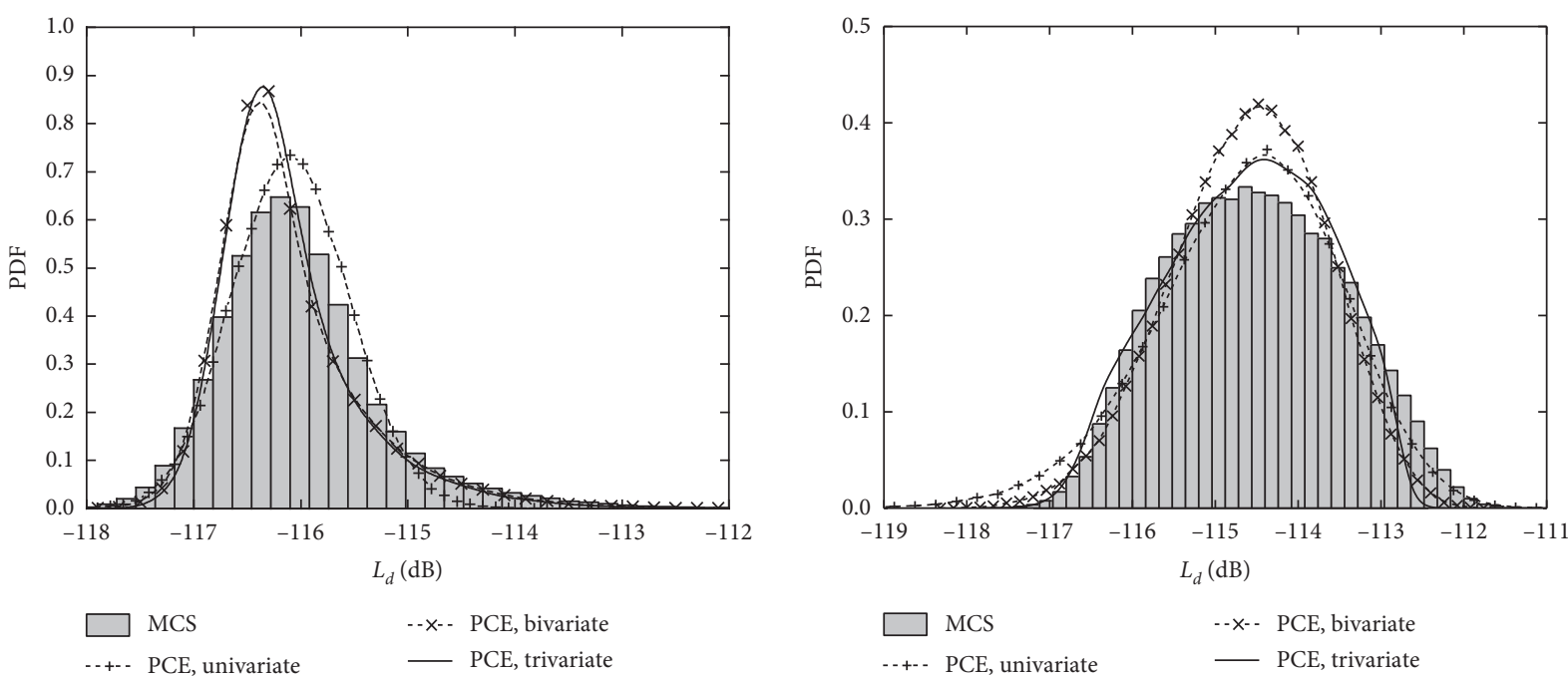

(c)

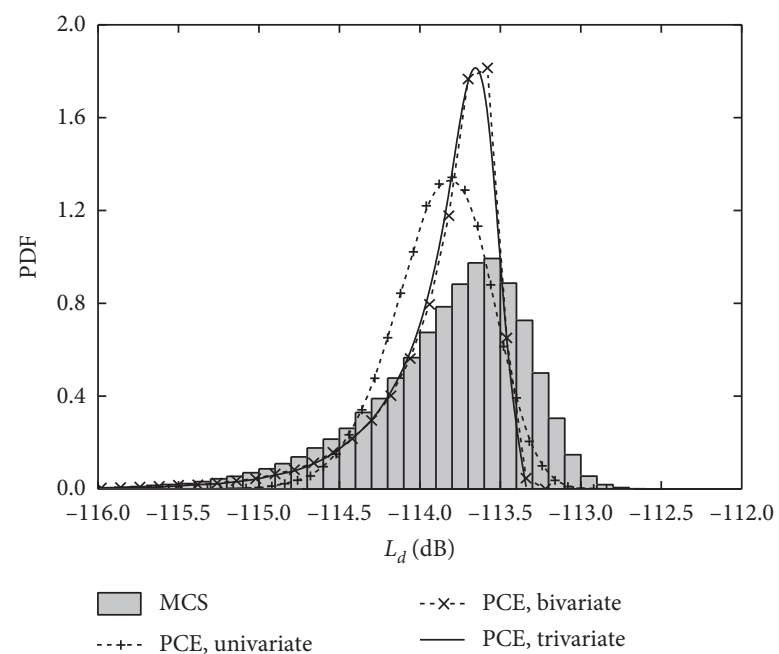

(e)

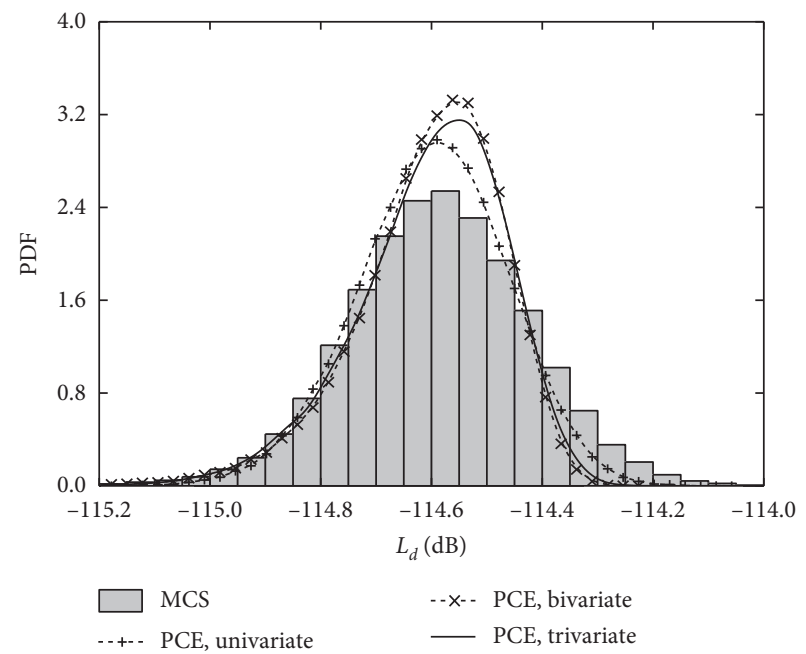

(f)

Figure 6: Continued. 


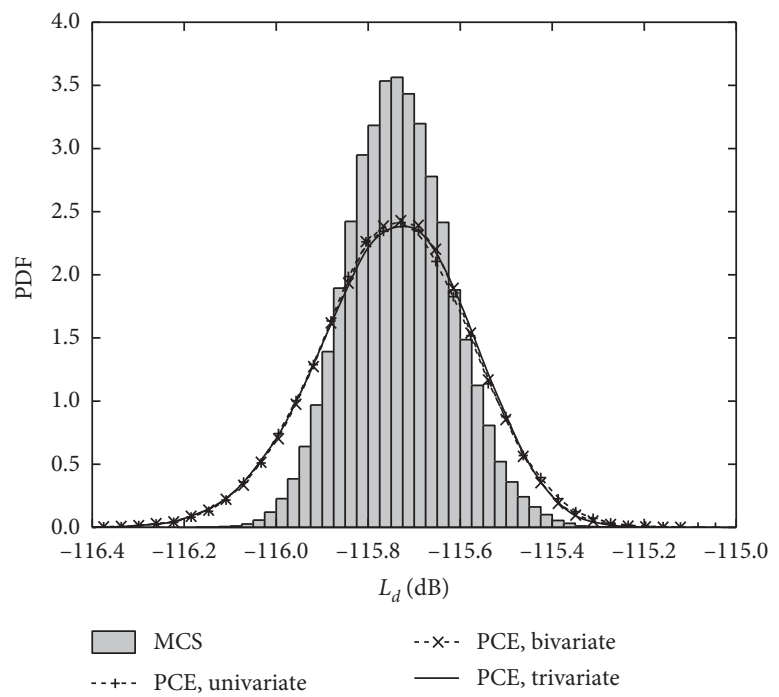

(g)

Figure 6: PDFs of the dynamic compliance amplitude within $29 \sim 35 \mathrm{~Hz}$. (a) $29 \mathrm{~Hz}$. (b) $30 \mathrm{~Hz}$. (c) $31 \mathrm{~Hz}$. (d) $32 \mathrm{~Hz}$. (e) $33 \mathrm{~Hz}$. (f) $34 \mathrm{~Hz}$. (g) $35 \mathrm{~Hz}$.

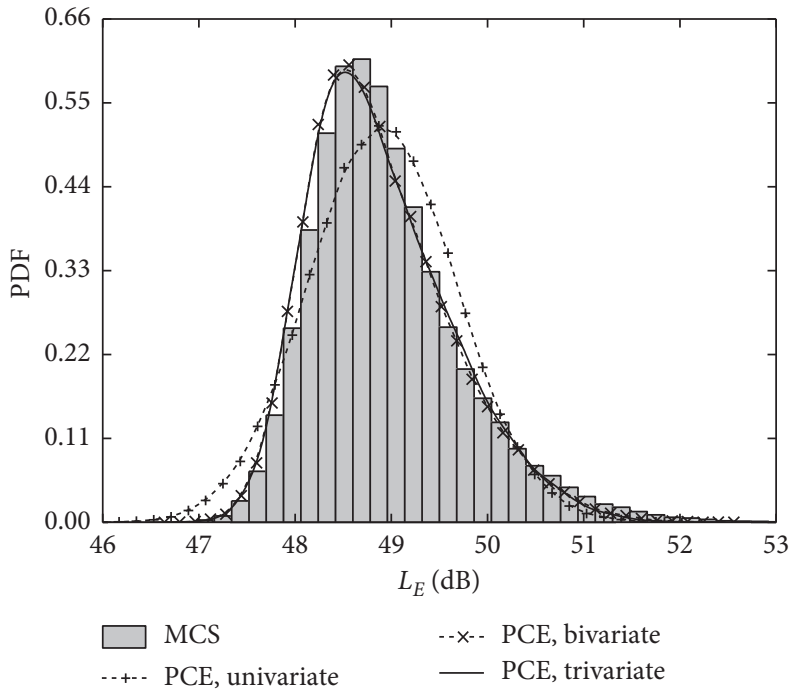

(a)

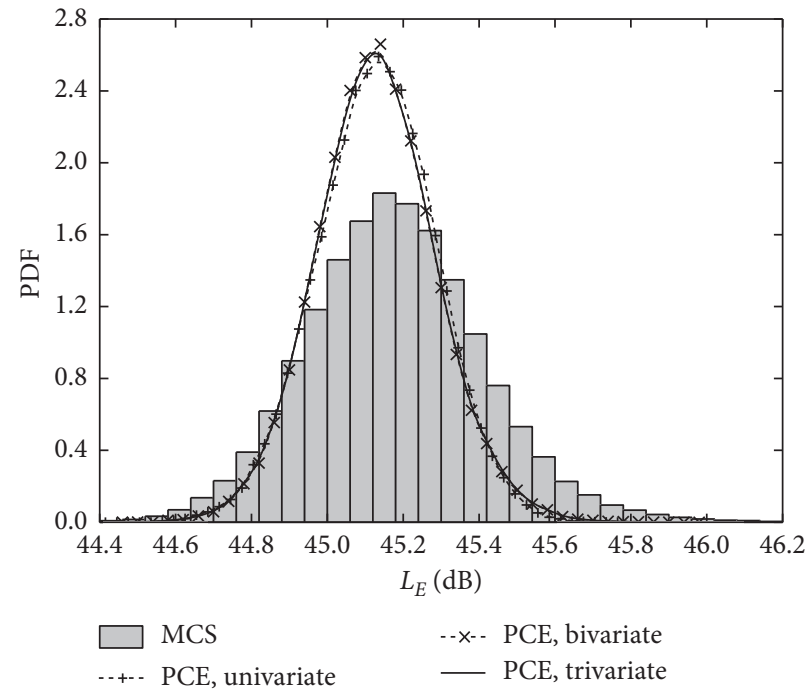

(b)

Figure 7: PDFs of the energy-level response of the $1 / 3$ Oct spectrum. (a) $f_{c}=20 \mathrm{~Hz}$. (b) $f_{c}=31.5 \mathrm{~Hz}$.

Figures 5 and 6 reveals that the PDFs have CSD. Random fluctuation of the resonant frequency leads to a large jump in the dynamic compliance amplitude when the mode damping is small; this leads to the PDFs having a larger local slope, causing a larger CSD. It is evident that increasing the mode damping is beneficial to eliminate CSD and, subsequently, to reduce its error.

3.3. Broad-Band Spectrum Analysis. In view of the platestructure FEM modeling shown in Figure 1, on the basis of narrow-band spectrum analysis, the vibration transmission and response of the $1 / 3$ octave (Oct) spectrum level are further analyzed using the proposed PCE approach.

(a) Figure 7 shows the PDFs of the $1 / 3$ Oct spectral vibration response with center frequency $f_{c}=20,31.5 \mathrm{~Hz}$. Results indicate that a higher $S$ variate approximation is better than the univariate one. There is no vertical vibration dominant mode (resonant frequency) within the frequency band of $20 \mathrm{~Hz}$, whose result is significantly better than that of the $31.5 \mathrm{~Hz}$ frequency-band result, which does have a vertical vibration dominant mode (resonant frequency). A comparison of Figure 7 with Figures 5 and 6 implies 


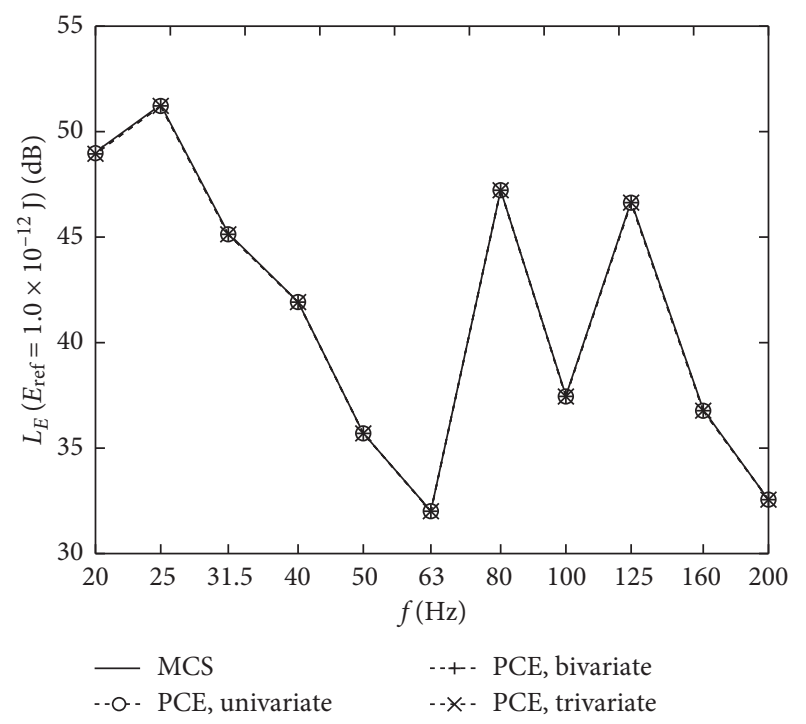

Figure 8: Mean of the energy-level response of the $1 / 3$ Oct spectrum.

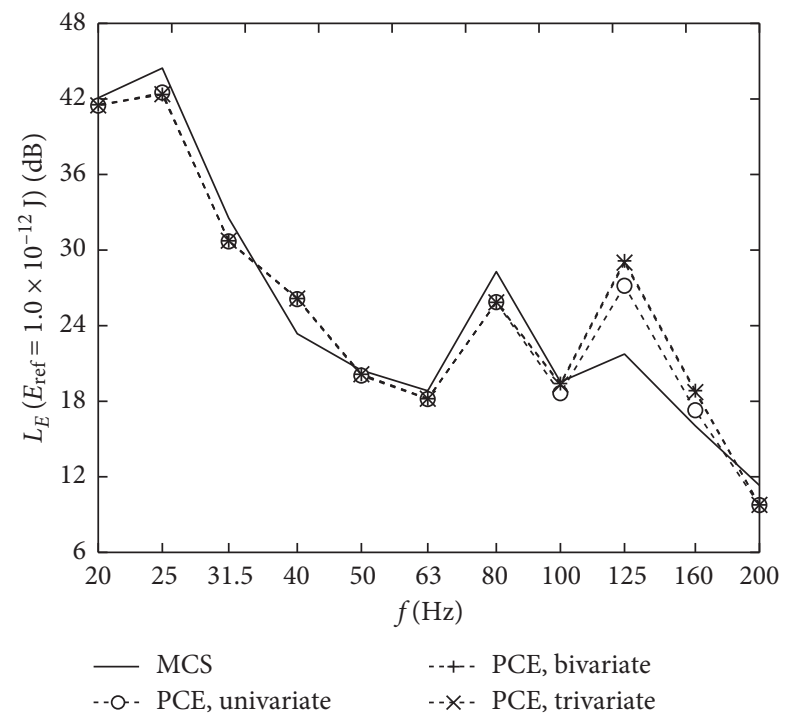

FIGURE 9: SE of the energy-level response of the 1/3 Oct spectrum.

that the PDF of a broad-band spectrum converges towards a Gaussian distribution, and the jump of the dynamic compliance amplitude is weakened due to the $1 / 3$ Oct broad-band energy superposition, which makes the proposed PCE approach more effective.

(b) Figure 8 shows the energy-level response mean of the $1 / 3$ Oct spectrum within the frequency range $[20,200] \mathrm{Hz}$. The curves in Figure 8 are almost identical, and their results coincide with equation (21), thus implying that the PCE approach is valid and feasible for the broad-band spectrum.

Correspondingly, Figure 9 shows the SE of the energy-level response of the $1 / 3$ Oct spectrum. Results show that, for the MCS, very small differences exist from the various PCE results; CSD behaves similarly, with similar reason, to the narrow-band spectrum. However, comparing Figure 9 with Figure 4, the relative error of the broad-band spectrum is significantly smaller than that of the narrow-band spectrum. This shows that the PCE approach for broad-band spectrum analysis is more efficient and accurate. Unfortunately, there is a certain degree of small error in the higher-order statistics, but it does not affect the accuracy of the PCE method.

\section{Concluding Remarks}

A vibration transmission analysis approach is presented based on the PCE of the random dynamic compliance for a system with uncertainties. The numerical illustrations demonstrated 
that the proposed PCE approach is efficient in comparison with MCS. Some conclusions were drawn as follows:

(a) Compared to the MCS, the results of narrow- and broad-band spectrum analysis under uncertain dynamical systems based on the proposed PCE approach are reasonable and feasible. The jump of the dynamic compliance amplitude caused by the random fluctuation of resonant frequency is weakened because of broad-band energy superposition.

(b) Compared to MCS, the PDF curves obtained by PCE have CSD, which is a relative error of vibration analysis. The error cannot be eliminated because of modal-modeling asymptotic estimation, even if we increase the order of statistics, multirandom variable decoupling, and Hermite polynomial truncation in the orthogonal polynomial expansion. In particular, random fluctuation of the resonant frequency is generated by the system uncertainty, causing a jump in the dynamic compliance amplitude.

(c) The estimated results will converge towards statistical energy analysis results along with an increase in mode overlap numbers. It is noteworthy that some important information about the narrow-band spectrum will be lost when carrying out broad-band spectrum analysis. Therefore, when applying the PCE approach to a random vibration system of lowmedium frequency, it is suggested that narrow- and broad-band spectrum analysis be implemented simultaneously.

However, the PDF estimation of the mode shape of the random vibration system, the jump in the dynamic compliance amplitude, and nonlinear problems need to be further investigated. Meanwhile, studies should focus on the PCE approach for the cross-point vibration transmission of the dynamical system. In addition, since the system is discretized into FEA models, whose boundary conditions of stochastic elastic constraints are described by finite number of stiffness, PCE can be used for random vibration analysis of the system in this study. However, the problem that PCE is computationally intractable, particularly for numerous random stiffness, even invalid for continuous elastic restraint, offers many challenges; therefore, the methodology of PCE also needs to be discussed in the future.

\section{Nomenclature}

$a, b$ : Order of the Hermite polynomial

$e: \quad$ Standard error level

$f: \quad$ Natural frequency

$f(x)$ : Marginal distribution density

$i, j$ : Node number

$k$ : Number of variables truncated

$l$ : Dimensional decomposition

$m$ : Number of Hermite polynomials

$n$ : $\quad$ Modal order

$q$ : $\quad$ Moments of arbitrary index

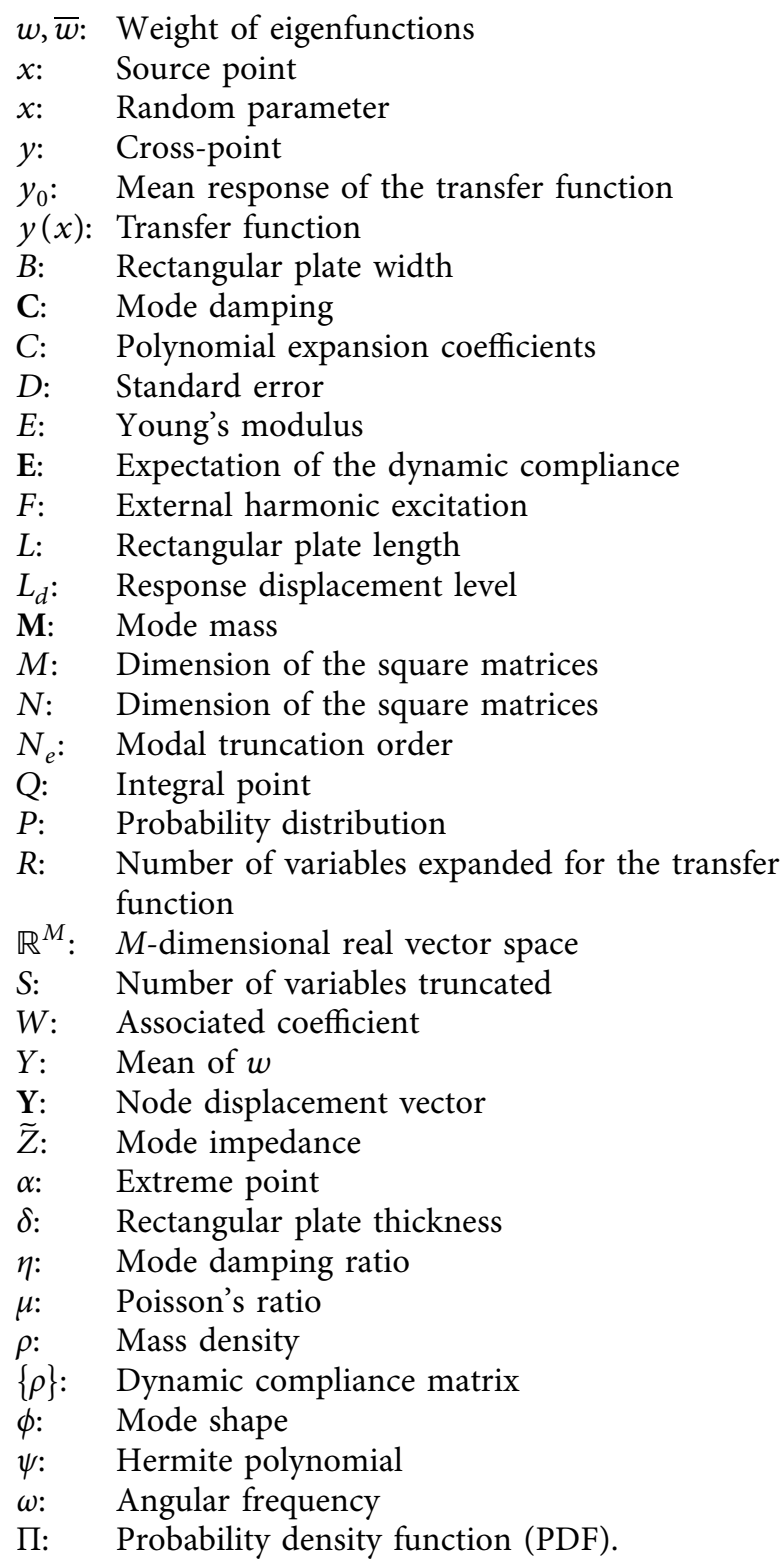

\section{Data Availability}

The data used to support the findings of this study are included within the article.

\section{Conflicts of Interest}

The authors declare no potential conflicts of interest with respect to the research, authorship, and/or publication of this article.

\section{Acknowledgments}

The authors gratefully acknowledge the support from the National Natural Science Foundation of China (Grant nos. 51475088 and 51705071) and Jilin Province Science and Technology Development Program of China (Grant nos. 20140101065JC, 20190201109JC, and 20190103058JH). 


\section{References}

[1] M. Shinozuka, "Monte Carlo solution of structural dynamics," Computers \& Structures, vol. 2, no. 5-6, pp. 855-874, 1972.

[2] M. Shinozuka and C.-M. Jan, "Digital simulation of random processes and its applications," Journal of Sound and Vibration, vol. 25, no. 1, pp. 111-128, 1972.

[3] B. Xiao, Z.-d. Lu, S.-x. Shi, C. Gao et al., "Volterra-series-based equivalent nonlinear system method for subharmonic vibration systems," International Journal of Systems Science, vol. 50, no. 3, pp. 479-494, 2019.

[4] B. Xiao, C. Gao, and Z. G. Liu, "Decoupling analysis on nonlinear system based on the modified generalized frequency response functions," Mechanical Systems \& Signal Processing, vol. 42, no. 1-2, pp. 283-299, 2014.

[5] T. Ye, G. Jin, Z. Su, and Y. Chen, "A modified Fourier solution for vibration analysis of moderately thick laminated plates with general boundary restraints and internal line supports," International Journal of Mechanical Sciences, vol. 80, pp. 29-46, 2014.

[6] T. Ye, G. Jin, and Z. Su, "Three-dimensional vibration analysis of laminated functionally graded spherical shells with general boundary conditions," Composite Structures, vol. 116, pp. 571-588, 2014.

[7] T. Ye, G. Jin, Z. Su, and X. Jia, "A unified Chebyshev-Ritz formulation for vibration analysis of composite laminated deep open shells with arbitrary boundary conditions," Archive of Applied Mechanics, vol. 84, no. 4, pp. 441-471, 2014.

[8] M. Kamiński and C. Wiley, The Stochastic Perturbation Method for Computational Mechanics, Wiley, Hoboken, NJ, USA, 2013.

[9] B. Huang, Q. S. Li, W. H. Shi, and Z. Wu, "Eigenvalues of structures with uncertain elastic boundary restraints," Applied Acoustics, vol. 68, no. 3, pp. 350-363, 2007.

[10] S. Q. Wu and S. S. Law, "Evaluating the response statistics of an uncertain bridge-vehicle system," Mechanical Systems and Signal Processing, vol. 27, no. 1, pp. 576-589, 2012.

[11] B. Xiao, Z. Sun, S. Shi et al., "On random elastic constraint conditions of Levinson beam model considering the violation of Saint-Venant's principle in dynamic," The European Physical Journal Plus, vol. 135, p. 168, 2020.

[12] J. Szafran, K. Juszczyk, and M. Kamiński, "Experiment-based reliability analysis of structural joints in a steel lattice tower," Journal of Constructional Steel Research, vol. 154, pp. 278-292, 2019.

[13] K. Handa and k Anderson, "Application of finite element methods in statistical analysis of structures," in Proceedings of the 3rd International Conference On Structure, Safety and Reliability Trondheim, pp. 409-417, Trondheim, Norway, June 1981.

[14] S. H. Chen, M. Song, and Y. D. Chen, "Robustness analysis of responses of vibration control structures with uncertain parameters using interval algorithm," Structural Safety, vol. 29, no. 2, pp. 94-111, 2007.

[15] S. Adhikari, "Joint statistics of natural frequencies of stochastic dynamic systems," Computational Mechanics, vol. 40, no. 4, pp. 739-752, 2007.

[16] S. Adhikari, "Matrix variate distributions for probabilistic structural dynamics," AIAA Journal, vol. 45, no. 7 , pp. 1748-1762, 2007.

[17] B. Pascual and S. Adhikari, "Hybrid perturbation-Polynomial Chaos approaches to the random algebraic eigenvalue problem," Computer Methods in Applied
Mechanics and Engineering, vol. 217-220, no. 3, pp. 153167, 2012.

[18] D. Sarsri, L. Azrar, A. Jebbouri et al., "Component mode synthesis and polynomial chaos expansions for stochastic frequency functions of large linear FE models," Computers \& Structures, vol. 89, no. 3-4, pp. 346-356, 2011.

[19] B. Xiao and Y. X. Li, "Statistical analysis of vibration response of plate structure considering parameter uncertainty," Journal of Vibration and Shock (Chinese), vol. 37, no. 9, pp. 52-57, 2018.

[20] K. Sepahvand, S. Marburg, and H.-J. Hardtke, "Stochastic free vibration of orthotropic plates using generalized polynomial chaos expansion," Journal of Sound and Vibration, vol. 331, no. 1, pp. 167-179, 2012.

[21] E. Bahmyari, M. R. Khedmati, and C. G. Soares, "Stochastic analysis of moderately thick plates using the generalized polynomial chaos and element free Galerkin method," Engineering Analysis with Boundary Elements, vol. 79, pp. 23-37, 2017.

[22] B. Najlawi, M. Nejlaoui, Z. Affi, and L. Romdhane, "Multiobjective robust design optimization of a sewing mechanism under uncertainties," Journal of Intelligent Manufacturing, vol. 30, no. 2, pp. 783-794, 2019.

[23] B. Najlawi, M. Nejlaoui, Z. Affi et al., "Multi-objective robust design optimization of the mechanism in a sewing machine," Mechanics and Industry, vol. 18, no. 6, p. 606, 2017.

[24] B. Najlawi, M. Nejlaoui, Z. Affi et al., "Multi-objective robust design optimization of a mechatronic system with uncertain parameters, using a polynomial chaos expansion method," Proceedings of the Institution of Mechanical Engineers, Part I: Journal of Systems and Control Engineering, vol. 231, no. 9, pp. 729-739, 2017.

[25] M. Hadigol and A. Doostan, "Least squares polynomial chaos expansion: a review of sampling strategies," Computer Methods in Applied Mechanics and Engineering, vol. 332, pp. 382-407, 2017.

[26] B. Xiao, W. Liu, C. Gao et al., "Source identification methods of low and medium frequency ship structural noise," Journal of Harbin Engineering University, vol. 36, no. 12, pp. 1596$1602,2015$.

[27] C. Monthus, "Statistical properties of the Green function in finite size for Anderson Localization models with multifractal eigenvectors," Journal of Physics A Mathematical General, vol. 50, 2017.

[28] P. R. Bevington and D. K. Robinson, Data reduction and error analysis for the physical sciences, McGraw-Hill, New York, NY, USA, 3 edition, 2003.

[29] S. Rahman, "A polynomial dimensional decomposition for stochastic computing," International Journal for Numerical Methods in Engineering, vol. 76, no. 76, pp. 2091-2116, 2008.

[30] H. Xu and S. Rahman, "A generalized dimension-reduction method for multidimensional integration in stochastic mechanics," International Journal for Numerical Methods in Engineering, vol. 61, no. 12, pp. 1992-2019, 2004.

[31] S. Shi, B. Xiao, G. Jin, and C. Gao, "Modeling and simulation of transverse free vibration analysis of a rectangular plate with cutouts using energy principles," Shock and Vibration, vol. 2018, Article ID 9609745, 16 pages, 2018. 